O27 (continued)

intervention would increase the availability of healthy foods in these food deserts.

Funding: University of Florida

\section{Influencing the Child Shopper with Coupons in Corner Stores: The CHOMPS Pilot Project}

Megan E. Lehnerd, MS, megan.lehnerd@tufts.edu, Tufts University, 150 Harrison Avenue, Boston, MA 02111; Anna R. McAlister, PhD, Michigan State University; Christina D. Economos, PhD, Tufts University; W. L. Adamowicz, PhD, FRSC, University of Alberta; Suzanne R. Howell, MPH, MS, Tufts University; Kaela Plank; Allison L. Gallop, MS, RD, CDE; Sean B. Cash, PhD

Objective: Evidence shows that children's independent food purchases in convenience stores are primarily purchases of energy-dense, nutrient-poor (EDNP) foods. Additional research is needed to understand how shifting children's snack food purchases could improve their diets.

Study Design, Setting, Participants, Intervention: This multi-disciplinary intervention utilizes economic, psychological, and nutritional insights to explore children's autonomous food purchase behaviors. Set in Somerville and Medford, MA, the intervention partnered with convenience stores near schools where kids shopped during out-of-school time. During our two-year, USDA-funded pilot, the Coupons for Healthier Options for Minors Purchasing Snacks (CHOMPS) study placed kids-only coupons in convenience stores with the goal of encouraging healthier snack purchases. Healthier snack coupons of varying discounts were paired with simple economic and health marketing messages.

Outcome Measures and Analysis: During before- and after-school times, data were recorded about the snack item, price, and other aspects of each purchase.

Results: Reported results include the observed purchase patterns of children, their responses to the coupon intervention in participating stores, and the nutritional composition of the observed purchases. Across more than 3,400 separate purchase events, we noted only modest coupon usage in the intervention phase. However, chip and candy purchases decreased, and the percentage of purchase events that included at least one targeted healthier food item more than doubled.

Conclusions and Implications: Results from this pilot indicate that kids-only coupons can assist with shifting snacking behavior outside of school settings. We anticipate that such an intervention would be even more impactful when combined with interventions focused on creating healthier corner stores - a strategy that is increasing in prominence.

Funding: USDA
029 Perspectives of Food Shoppers at Convenience Stores in Navajo Nation Food Deserts

Emily Piltch, MPH, BA, BS, emily.piltch@tufts.edu, Tufts University, 55 Los Gatos Circle, Sacramento, CA 95831; Tim Griffin, PhD, Tufts University; Robert F. Houser, PhD, Tufts University; Sonya Shin, MPH, MD, Community Outreach and Patient Empowerment (COPE); Carmen George, MS

Objective: Navajo face a double-burden of high rates of chronic disease and food insecurity, but little is known about the role small stores on the Navajo Nation play in providing healthy, affordable food. This study sought to understand barriers to and facilitators of purchasing produce at these stores.

Study Design, Setting, Participants: July to September 2016, a convenience sample of 72 shoppers participated in brief interviews outside eleven small stores in the New Mexico region of Navajo Nation.

Outcome Measures and Analysis: Shoppers discussed their shopping patterns, preferences and also food availability at the small store.

Results: Most participants (64\%) shopped primarily at bigbox stores outside their communities, yet about half shopped at the small store at least twice per week. The most common reasons for shopping at the small store were convenience to home/work and fuel purchase. The most commonly purchased items were snacks (42\%), staples (26\%), and soda (25\%). Almost half of participants worried in the last month about having enough food to feed their families and said that at least one person in the household participated in a federal assistance program. Shoppers would be more inclined to purchase produce if a greater variety of produce were available at affordable prices.

Conclusions and Implications: While small stores are the only retail options close to home for many Navajo, purchases are primarily snacks, rarely produce. The current and potential roles of small stores in food purchasing behaviors in rural communities will be discussed. This work provides basis for supporting innovative, community-based strategies for increasing access to healthy food close to home.

Funding: USDA

\section{Use of the Socioecological Model to Identify Factors of an Obesogenic Environment in Families with Young Children in Mexico}

Samantha Ramsay, PhD, LDN, RD, sramsay@uidaho.edu, University of Idaho, 875 Perimeter Drive, MS 3183, Moscow, ID 83844-3183; Jennie Davis, MS, University of Idaho; Lauren Keeney, MS; Samantha Worden, MS; Siew Guan Lee, RD, MS; Mackenzie Ferrante, MS, RD, Colorado State University; Jocelyn Macias, LN, Universidad Autónoma de Guadalajara; Delores Loaiza Lizarraga, PhD, $R D$

Objective: To identify factors related to an obesogenic environment in a small sample of families with young

Continued on page $S 13$ 